\title{
A PSYCHOL example
}

\author{
ROBERT S. MCLEAN \\ Department of Computer Applications \\ The Ontario Instinute for Studies in Education \\ 252 Bloor Street W'est. Toronto. Ontario. Canada M.SS I I't
}

PSYCHOL, a computer language for controlling psychological experiments, has been described in detail elsewhere in this journal (McLean, 1969). The reader is referred to that article for a more complete description of the features of the language. This paper presents a PSYCHOL program to control the Sternberg (1966) experiment, to allow the reader to compare PSYCHOL with the other languages discussed at the symposium.

PSYCHOL was the first attempt to provide ways of expressing the control of a psychological experiment within a general-purpose computer language. The language was created at the Computer-Controlled Psychological Laboratory (CCPL) at Carnegie-Mellon University in 1967-1968. It ran on a time-shared Honeywell DDP-116 computer used to control psychological experiments. The time-sharing system was created by the CCPL staff and was capable of running up to eight separate experiments simultaneously. The input/output devices were connected by way of a generalized 16-bit interface; video-based alphanumeric displays were also available.

PSYCHOL is based on additions to the general-purpose programming language, ALCOL 60. ALGOL was selected as the base language because it was the language of choice at Carnegie-Mellon University at the time and was widely known among people in psychology and computer science there. In addition, it offers such interesting features as its inherent block structure. mnemonic identifiers and labels, and the ability to make compound statements.

\section{THE EXAMPLE PROGRAM}

Many of the features added to ALGOL 60 are illustrated by the sample program shown in Fig. 1. This program controls a human memory experiment in which a list of up to six single digits is presented, followed by a probe digit that may or may not be in the set presented. The $S$ is required to push one of two buttons to indicate whether the probe was in the set or not. The meaning of the buttons is assigned randomly on each trial by giving the $S$ an indication of the button to use for the positive case just prior to the presentation of the probe. There are a total of 168 trials, including 24 practice trials.

The format of the program in Fig. 1 is arbitrary, and multiple spaces are used only for clarity on the printed page. Each statement in PSYCHOL ends with a semicolon; a statement beginning with the word COMMENT has no effect. The several statements between a BEGIN and END constitute a compound statement.
In the sample program, a declaration is used to define the stimulus list to contain the numbers 1 to 10 , the last value displaying a zero on the particular display device. The RESPONSE declaration sets up the translator to expect response input to come from the experimental room via the 16-bit interface: whenever the identifier "RESP" is used, its value will be the input from the room interface.

Identifiers (variables) can have any number of characters: in addition, periods can be included to improve their readability. Thus, TRIAL.NO is a variable that records the number of trials that occur. The identifiers TRIAL and LOOP are labels in the program.

PERMUTE randomizes the list STIM.LIST; INITIALIZE establishes a pointer to its first element. RANDOM is a function that returns a value over the range 0 to $n-1$; thus, SIZE is set to the length of list for this trial.

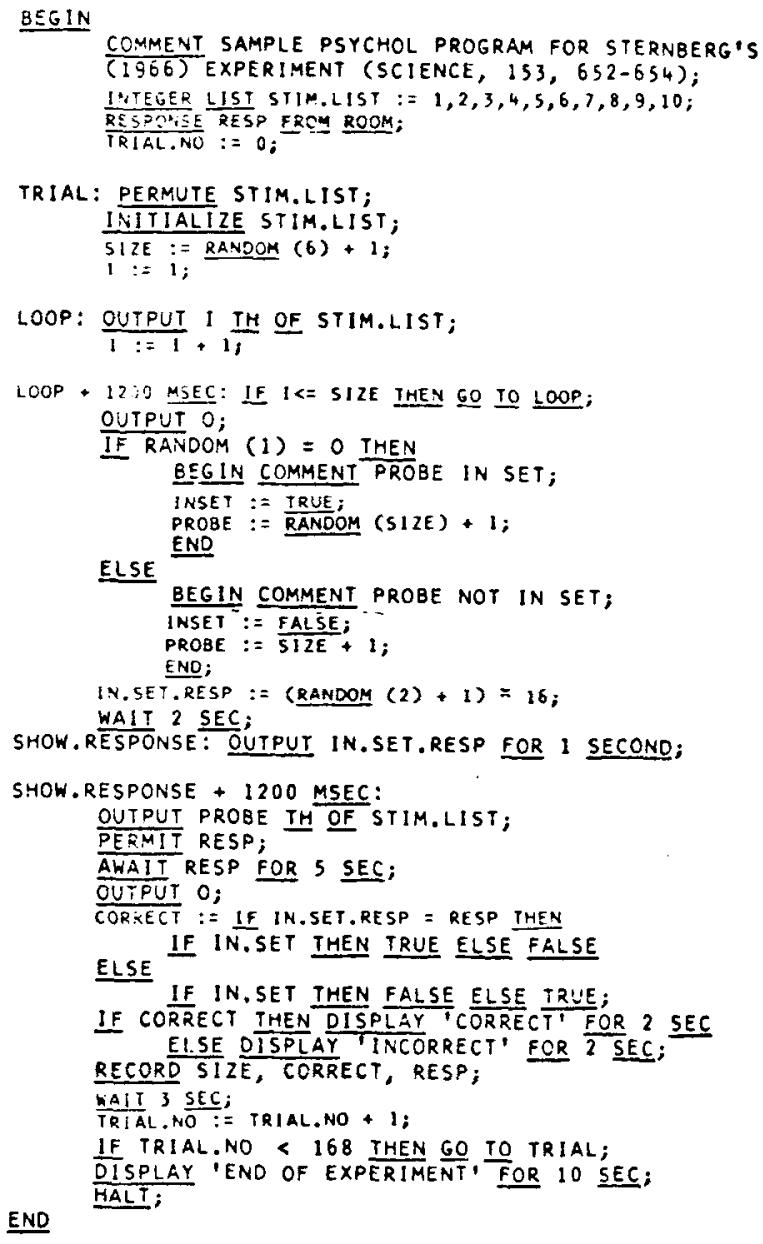

Fig. 1. 
The three statements beginning at LOOP present the list of stimuli. one each $1.2 \mathrm{sec}$. The OUTPUT command directs the value determined by evaluating the expression (I TH OF STIM.LIST) to the experimental room via the generalized output interface; in this case, the $\mathrm{S}$ is viewing a display device connected to the general output interface. LOOP +1200 MSEC is an example of a temporal label; this feature suspends execution of the program until the required time has elapsed since last passing the reference label-LOOP in this case.

OUTPUT $O$ turns off the display. The conditional statement determines, with equal probability, whether the probe is to be taken from the set or not, and sets a flag, IN.SET, to record that fact. The variable IN.SET.RESP is then set to select randomly a response the $S$ is to use if he believes the probe to be a member of the set.

After a 2-sec wait, the $S$ is informed of the response to be made to indicate set membership. This is displayed for $1 \mathrm{sec}$; this is an example of a command that is automatically cancelled following the expiration of its duration. When $1.2 \mathrm{sec}$ have elapsed following the onset of the set membership response display, the probe digit is output. At this point, the response equipment is enabled with the PERMIT RESP statement. allowing the $S$ to respond. He is given up to $5 \mathrm{sec}$ to respond, execution of the program being held at that point until he responds or the time expires. Upon either condition, the display is turned off. The variable CORRECT is set to TRUE or FALSE. depending upon the response and the correct response. Then the word "CORRECT" or "INCORRECT" is displayed as feedback to the $S$ for $2 \mathrm{sec}$. Information concerning the size of list, the S's response, and its correctness is recorded for later analysis.

Three seconds later, the experiment continues with the next trial, unless 168 trials have occurred. In that case, "END OF EXPERIMENT" appears on the video display for $10 \mathrm{sec}$ and the experiment is concluded.

\section{CURRENT STATUS}

PSYCHOL was implemented in 1968 on the CCPL computer. That implementation depended upon particular bulk storage equipment that later became unserviceable. A generalized compiler for PSCYHOL was begun in 1969 which would produce object code for a number of computers. This project was never completed, and the language is currently inactive.

\section{REFERENCES}

McLean, R. S. PSYCHOL: A computer language for experimentation. Behavior Research Methods \& Instrumentation, $1969,1,323-328$.

Sternberg, S. High-speed scanning in human memory. Science, $1966,153,652-654$

\section{SCAT: Design criteria and software*}

\author{
PETER G. POLSON† \\ The Computer Laboratory for Instruction \\ in Psychological Research \\ University of Colorado, Boulder, Colorado 80302
}

SCAT is a high-level language for writing programs that control experimental procedures and record data. This paper discusses general design criteria for higher level languages and the basic structure of the SCAT language. A second section of the paper briefly describes the compiler and gives a description of the operating system.

Extended State Change Algorithm Translator (EXTENDED SCAT) is a language that is used to describe experimental procedures involving contingencies between discrete sets of stimuli and responses, e.g.. operant paradigms, concept identification, two-person games, etc. We have been using SCAT at the Computer Laboratory for Instruction

*The development of the EXTENDED SCAT system was supported in part by National Science Foundation Grant GJ 453 to D. E. Bailey.

tRequests for reprints and more detailed information about the EXTENDED SCAT system should be sent to Peter G. Polson, CLIPR, 1511 University Avenue, University of Colorado, Boudder, Colorado 80302 . in Psychological Research for the last 2 years to teach real-time programming concepts to undergraduate and graduate students and to write programs that run experiments. The SCAT language was originally developed by the Grason-Stadler Company as part of a real-time system based on a DEC PDP-8/I. EXTENDED SCAT is a downwardly compatible dialect. Our additions to the SCAT language enable us to exploit the features of a medium-scale machine like the Xerox Sigma 3 or the IBM 1800 and to program a wider range of experimental paradigms.

This paper discusses principles underlying the structure of the SCAT language in the context of general criteria for special-purpose programming languages (Sammet. 1969). The EXTENDED SCAT compiler is briefly described, and the operating system is described in detail. Only a short outline of the SCAT language itself is included in this paper. See Polson (1971) or Polson \& Campbell (1972) for a complete description of EXTENDED SCAT

\section{DESIGN CRITERIA AND THE STRUCTURE OF SCAT}

Special-purpose programming languages. e.g.. SCAT. ACT. PSYCHOL. etc. have two functions. First. suth 